Research Article

\title{
Clinical Application of Three-Dimensional Reconstruction Technique in Thoracoscopic Pneumonectomy
}

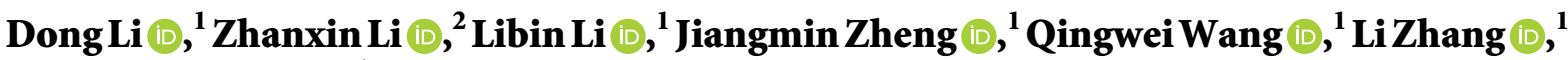 \\ and Zhigang Guo ${ }^{1}{ }^{1}$ \\ ${ }^{1}$ Surgery Development I of the General Hospital of Jizhong Energy Xingtai Mining Industry Group Co., Ltd., Xingtai, \\ Hebei 054000, China \\ ${ }^{2}$ Respiratory Department of Hebei General Hospital for Veterans, Xingtai, Hebei 054000, China
}

Correspondence should be addressed to Zhigang Guo; 15140621150718@post.eurasia.edu

Received 20 June 2021; Accepted 8 September 2021; Published 22 September 2021

Academic Editor: Gustavo Ramirez

Copyright ( 92021 Dong Li et al. This is an open access article distributed under the Creative Commons Attribution License, which permits unrestricted use, distribution, and reproduction in any medium, provided the original work is properly cited.

Objective. To investigate the clinical value of 3D computed tomography bronchoangiography (3D CTBA) in thoracoscopic anatomical lung segment resection or combined lung segment resection. Methods. The clinical data of 25 patients with non-smallcell lung cancer who underwent thoracoscopic radical lobectomy and pulmonary segmentectomy with pulmonary nodules were retrospectively analyzed. All patients underwent preoperative thin-slice CT scan, and the bronchus, pulmonary artery, and pulmonary vein were reconstructed by DeepInsight software. Meanwhile, pulmonary nodules, tumors, or enlarged lymph nodes were reconstructed. Accurate preoperative planning was carried out through preoperative reconstruction of three-dimensional images, especially the variation of pulmonary bronchus and blood vessels, the relationship between tumors and enlarged lymph nodes and pulmonary blood vessels, and the precise positioning of pulmonary nodules in pulmonary segments. Compared with preoperative three-dimensional reconstruction, intraoperative real-time navigation can achieve accurate operation. The intraoperative conversion to thoracotomy, operative time, intraoperative bleeding and postoperative hospitalization time, drainage tube removal time and total drainage volume, and the incidence of perioperative complications were recorded. Results. The anatomical structure and variation of bronchus and pulmonary vessels were clearly reconstructed in all patients, and the reconstruction of the relationship between central tumor and enlarged lymph nodes and blood vessels was satisfactory. The location of pulmonary nodules in pulmonary segments was clearly defined, and preoperative planning was performed accurately. All patients underwent real-time intraoperative navigation, and precise surgery was performed according to the preoperative planning. The operation was successfully completed without any transfer to thoracotomy or intraoperative accidental bleeding. The operative time was $(147.60 \pm 37.77) \mathrm{min}$, the intraoperative blood loss was $(33.82 \pm 22.17) \mathrm{mL}$, the postoperative hospital stay was $(7.02 \pm 1.78) \mathrm{d}$, drainage tube removal time was $(4.68 \pm 1.60) \mathrm{d}$, and postoperative total drainage volume was $(221.00 \pm 135.03)$ $\mathrm{mL}$; there were no severe complications and no death during perioperative period. Conclusion. The application of three-dimensional reconstruction technique for preoperative evaluation and subsequent thoracoscopic pulmonary segmental resection can achieve accurate, safe, and effective pulmonary segmental resection, reduce the difficulty of thoracoscopic pulmonary segmental resection, reduce the risk of surgery, and improve the surgical effect.

\section{Introduction}

In recent years, an increasing number of asymptomatic ground glass nodules (GGNs) with diameter $\leq 2 \mathrm{~cm}$ have been found in physical examination. Anatomic segmental pulmonary resection is an important surgical method for the treatment of pulmonary nodules. Anatomic segmental pulmonary resection can completely remove the target lesion, minimize lung tissue damage, and ensure the structural integrity and normal function of adjacent lung segments on the premise of negative surgical margin [1]. Due to the complex anatomical structure of lung segments and the wide variety of segmental/subsegmental bronchial and vascular variations, the difficulty of pulmonary segmental resection 
varies greatly, so it is very difficult to achieve thoracoscopic anatomical pulmonary segmental resection for relatively complex lung segments. The wide application of 3D CT bronchography and angiography (3D CTBA) provides a powerful technical support for anatomical segmentectomy [1]. Preoperative 3D imaging technology can accurately convert patients' CT images into 3D images, which can effectively solve the technical difficulties of pulmonary segmental resection, reduce the difficulty of surgery, and improve the treatment effect.

Lung cancer is now at the top of the list of cancers. Lowdose spiral CT can detect more patients with early lung cancer. Surgery has become the preferred treatment for patients with early lung cancer. At present, thoracoscopic lobectomy has been widely used as a standard operation in clinical practice, but for patients with small pulmonary nodules, lobectomy may cause significant lung function injury and affect postoperative quality of life, especially for patients with poor preoperative lung function or elderly patients. Driven by the concept of precision treatment, pulmonary segmental resection or combined pulmonary segmental resection has become a research hotspot in recent years [2]. Accurate pulmonary segmental resection can not only completely remove small pulmonary nodules and ensure negative surgical margin but also retain more effective lung function, which is conducive to rapid postoperative recovery of patients. Therefore, it is particularly important to carry out precise pulmonary segmentectomy under the principle of ensuring oncology. Three-dimensional reconstruction can clearly locate small pulmonary nodules from stereoscopic vision, predict the resection range, and find the abnormal blood vessels and bronchus, making the operation more accurate and safe. This study retrospectively analyzed the significance of preoperative three-dimensional reconstruction in thoracoscopic pulmonary segmental resection or combined pulmonary segmental resection, in order to provide data for clinical practice.

\section{Materials and Methods}

2.1. General Information. The clinical data of 50 patients with non-small-cell lung cancer who underwent thoracoscopic lobectomy and pulmonary segmentectomy with pulmonary nodules in the Department of Thoracic Surgery of the First Affiliated Hospital of Bengbu Medical College from November 2018 to December 2019 were retrospectively analyzed. There were 18 males and 32 females (aged (56.60 \pm 10.16$)$ years old). There were 37 cases of pulmonary lesions, 4 cases of blood in sputum, 4 cases of chest tightness, and 5 cases of irritant dry cough. Thin-slice CT scan of chest was performed before surgery, and there were no contraindication evidences for lung function, electrocardiogram, lung cancer-related examination, or routine examination. Thoracoscopic radical lobotomy was performed in 25 cases, including 8 cases of right upper lung cancer, 2 cases of right middle lung cancer, 9 cases of right lower lung cancer, 3 cases of left upper lung cancer, and 3 cases of left lower lung cancer. Thoracoscopic segmentectomy was performed in 25 patients with pulmonary nodules, and 57 lung lesions were removed. All patients with lung cancer lobectomy met the indications of radical surgery. Pulmonary segmentectomy meets the following requirements: (1) lesion diameter $\leq 2 \mathrm{~cm}$ and meets one of the following conditions: ground glass composition $\geq 50 \%$, rapid pathology suggested carcinoma in situ or microinfiltrating carcinoma, and doubling time $\geq 400$ days; (2) comfortable surgery with multiple nodules in one or both lungs and need to preserve more lung function; and (3) benign lesions that cannot be wedge excised due to location, size, and other reasons. The development of this technology has passed the ethical review and high-tech support of the First Affiliated Hospital of Bengbu Medical College.

\section{Methods}

3.1. Precise Preoperative Planning. Thin-slice CT scan data of the chest were collected. The optimal data requirements were as follows. (1) Thin-slice CT of the chest with thickness $<1 \mathrm{~mm}$ was collected with equipment above 64 slice CT machine. (2) Inhale deeply and scan while holding breath. (3) The dose and time of contrast agent should be controlled for enhanced CT. The "time-density curve" was obtained by group injection test. After analysis, the injection dose and delayed scanning time of contrast agent were set. (4) Avoid joint scanning with head, neck, and abdomen, avoid repeated scanning, and remove foreign bodies in the scanning area. CT image information is saved in DICOM format for application. The DeepInsight 3D reconstruction software authorized by Shenyang Neusoft Medical System Co., Ltd., was applied. The reconstruction methods were as follows. (1) The chest CT $2 \mathrm{D}$ image information was transferred into the DeepInsight system in DICOM format. (2) By image loading processing, trachea threshold calculation, extraction of trachea, blood vessels, lymph nodes, pulmonary nodules, and lung masses, blood vessel staining, and other steps, the bronchus, arteriovenous vessels, and lymph nodes were reconstructed. (3) To segment lymph nodes, lung masses, or nodules from branchial trachea and pulmonary arteriovenous vessels and to evaluate the branching, diameter, shape, and variation of anatomical structures such as bronchi, pulmonary artery, and pulmonary vein, the three-dimensional spatial adjacent relationship and invasion degree of lymph nodes, pulmonary nodules, and lung masses with bronchus, pulmonary arteries, pulmonary veins, and other structures were assessed, and the spatial location of pulmonary segments where pulmonary nodules were located was accurately positioned for accurate preoperative planning.

3.2. Surgical Methods. All patients were treated with combined inhalation anesthesia. The operation was performed under the condition of single-lung ventilation through double-cavity air tube intubation or occlusion tube. The lateral decubitus position was performed with single hole or single operator hole. According to the development of pulmonary fissure and the presence of pulmonary vascular invasion, the surgical sequence was one-way or traditional. According to the preoperative reconstruction of three- 
dimensional images, the bronchus, incomplete pulmonary fissure, pulmonary artery and vein, and the intersegmental plane were accurately dissected and treated to avoid injury and misbroken bronchus and pulmonary artery and vein. Systemic lymph node dissection was performed at the same time for radical resection of lung cancer, and lymph node sampling was performed at the same time for segmental surgery. After the operation, the anesthesiologist was asked to dilate the lungs to check the air leakage and whether suture reinforcement was needed. During the operation, the image fusion method was used in the same step as the operation. The model was rotated and transparent under the assistant platform to synchronously make the 3D reconstruction image consistent with the intraoperative anatomical structure. Real-time verification and navigation were performed during nodular positioning, pulmonary arteriovenous and bronchial treatment, and other operations to ensure accurate operation. All patients underwent surgery successfully, and no cases were transferred to thoracotomy (see Figures 1 and 2).

3.3. Clinical Observation Data. Record three-dimensional reconstruction of preoperative planning and postoperative usage of intraoperative navigation and operation method, operation time, intraoperative blood loss, postoperative urethral closed chest drainage tube time and total flow rate and postoperative hospital stay, etc., and the postoperative complications such as lung staining, BPF, bleeding, respiratory failure, and severe liquid pneumothorax were observed and recorded.

\section{The Results}

4.1. Preoperative Planning and Use of Intraoperative Navigation for 3D Reconstruction. All patients could accurately complete the preoperative plan, clearly reconstruct the anatomical structure of branch trachea and pulmonary vessels, and were satisfied with the reconstruction of the relationship between central tumor and enlarged lymph nodes and vessels. They could accurately define the position relationship of pulmonary nodules in the pulmonary segment and carry out accurate preoperative planning. All patients underwent realtime intraoperative navigation, and the operation was performed according to the preoperative plan, and the operation was successfully completed.

4.2. Surgical Methods under the Guidance of $3 D$ Reconstruction Technology. All patients underwent thoracoscopic radical lobectomy and systemic lymphadenectomy in 25 cases, including right upper lobectomy in 16 cases, right middle lobectomy in 4 cases, right lower lobectomy in 9 cases, left upper lobectomy in 3 cases, and left lower lobectomy in 6 cases. 50 patients underwent thoracoscopic segmental resection with lymph node sampling, including 27 patients with single segmental lung, 13 patients with combined segmental lung or subsegmental lung, and 14 patients with combined segmental lung wedge. A total of 57 lung lesions were resected.
The internal and peripheral vascular display rate of the tumor was significantly higher than that of MSCT enhanced scanning, which was significantly different $(P<0.01$ or 0.05$)$. MPR technology showed high morphological characteristics of pulmonary nodules, as shown in Table 1.

\section{Discussion}

The morbidity and mortality of lung cancer are in the forefront of malignant tumors, which poses a serious threat to human health and life. With the popularization of doublelung CT screening program, more and more small pulmonary nodules with diameter $\leq 2 \mathrm{~cm}$ can be found, among which GGNs account for a large proportion, and these nodules have a certain malignant probability, among which the proportion of atypical adenomatoid hyperplasia, adenocarcinoma, microinfiltrating adenocarcinoma, and infiltrating adenocarcinoma are relatively high [3]. It is a problem that every thoracic surgeon needs to consider and solve to preserve lung tissue and protect lung function to the greatest extent while ensuring the radical effect of surgery. Studies have shown that there is no significant difference in overall survival rate between lobectomy and sublobectomy in stage Ia patients with tumors $<2 \mathrm{~cm}$, suggesting that precise anatomical segmental pulmonary resection is an important surgical method for treating pulmonary nodules. At present, complete thoracoscopic pulmonary segmentectomy has been widely used in the treatment of pulmonary nodules in tertiary hospitals, but it has not been widely used in primary hospitals. The main reason is that the anatomical structure of lung segment is complex, and the variation of segmental trachea and blood vessels is diverse. The incidence of different types of segmental trachea and blood vessel variation is still lack of specific data and database for query.

Because ordinary CT scan is a two-dimensional display image, sometimes it is difficult to accurately locate the pulmonary segment where the pulmonary nodules are located and the arteriovenous direction of the target segment; especially when the nodules are located at the intersegment junction, the localization is even more difficult. It is often difficult for ordinary CT to identify the mutated intersegmental vessels, which increases the risk and difficulty of surgery. Three-dimensional computed tomography bronchoangiography (3D CTBA) can accurately distinguish and mark the pulmonary artery, pulmonary vein, bronchus, and pulmonary nodules through software and conduct multilevel and multiangle observation and judgment on the reconstructed images, so as to set the preoperative plan in advance and select the optimal surgical path and operation process. 3D reconstruction software is based on preoperative high-resolution enhanced CT reconstruction, so the quality of CT results directly affects the accuracy of reconstruction. High-resolution CT can be adjusted with a minimum node thickness of $0.5 \mathrm{~mm}$, but a lower scan thickness will result in a longer scan time and radiation dose. Secondly, the deeper breathing and longer breath-holding time of the patient during the examination can make the final bronchiole aeration more obvious and improve the accuracy of tracheal reconstruction. Enhanced CT images are best captured in the 

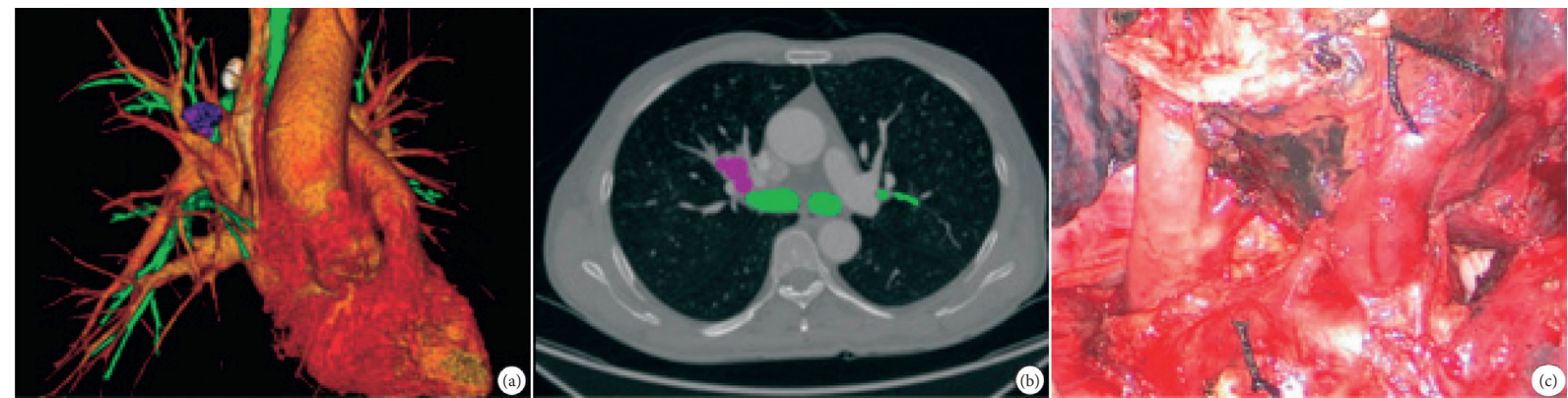

FiguRE 1: 3D reconstruction and intraoperative images of right upper lung central lung cancer. (a) 3D reconstruction picture. (b) Cross section of the chest mass on enhanced CT. (c) Intraoperative images.
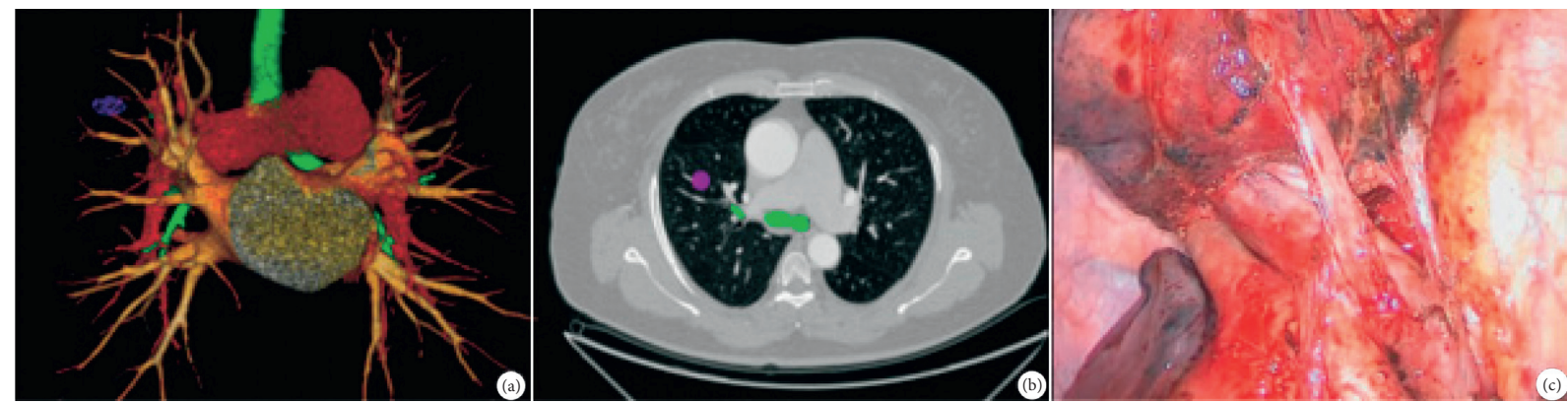

FIGURE 2: 3D reconstruction and intraoperative image of right upper anterior segment lung resection. (a) 3D reconstruction picture.

(b) Thin-slice high-resolution chest CT. (c) Intraoperative images.

TABLE 1: Comparison of 115 SPN image displays (\%).

\begin{tabular}{lccc}
\hline Morphological characteristics & MSCT enhancement scanning & MPR technology & $P$ value \\
\hline Leaf differentiation characteristics & $42(36.52)$ & $58(50.43)$ & $<9(60.00)$ \\
Vascular cluster characterization & $39(33.91)$ & $40(34.78)$ & $<0.05$ \\
Burr-spur characterization & $42(36.52)$ & $78(67.83)$ & $>0.01$ \\
Pleural retraction & $38(33.04)$ & $52(45.22)$ & $<0.05$ \\
Air bronchial characteristics & $25(21.74)$ & $36(31.30)$ & $<0.01$ \\
Empty bubble characteristics & $19(16.52)$ & $<0.01$ \\
\hline
\end{tabular}

arterial phase so that they can be more clearly distinguished from the veins. The authors' experience was as follows: preoperative high-resolution CT thickness was controlled within $0.5 \sim 1 \mathrm{~mm}$, with the highest $<1.5 \mathrm{~mm}$; preoperative detailed information was given to the patient for cooperation requirements; and image capture was performed in the pulmonary artery phase.

The trachea is the core of software reconstruction. The software reconstruction of trachea is based on the air in the trachea, so there is an error of $1-4 \mathrm{~mm}$ thickness of the airway wall between the reconstruction results and the real situation. If the patient does not hold his breath during the scan, a remodeling of the distal trachea may occur. At this point, you need to make up manually. Similarly, when using software to reconstruct blood vessels, there may be confusion in some small arteries and veins, which requires careful identification. Each software has its own advantages in the reconstruction. At present, the Mimics software is widely used and easy to obtain. It has been updated to the 220 version. Mimics, InCool, and other software use intersegmental vein to divide lung segments, while IQQA software uses trachea as the core for intersegmental plane division. Due to the fact that lung segments are three-dimensional conical, part of lung segments is not separated by a straight line. In more cases, adjacent lung segments are interlaced with each other, and the broken line boundary will occur when searching for the intersegment plane by means of ventilation collapse, fluorescence, pure oxygen lung expansion, etc. Therefore, the reconstruction results of software cannot fully reflect the division of intersegment plane, and clinicians need to comprehensively consider the reconstruction results and intraoperative intersegment boundaries. Compared with the vein, the variation of pulmonary segment artery is smaller, and its shape is accompanied by the trachea, which can be used to verify each other during reconstruction and surgery. If the pulmonary segment vein is too much, there may be symptoms of hemoptysis after surgery. In pulmonary segmentectomy, intersegmental vein and intersegmental plane can be mutually verified. After severing the main intersegmental vein branches, the treatment of the remaining intersegmental vein branches can be weakened. The clinician also needs to 
pay attention to the transition between the intrasegmental and intersegmental veins. Intersegmental veins at the time of segmental resection may change their role to intrasegmental veins at the time of combined segmental or combined subsegmental resection. After 3D CTBA reconstruction, according to the direction of tracheal blood vessels, clinicians can review the surgical target area again according to the reconstruction results and design the preoperative plan more accurately.

Compared with lobectomy, anatomic segmental or wedge resection preserves more lung function and reduces the incidence of postoperative respiratory failure. For pulmonary nodules with superficial subpleural surface and easy location, wedge resection is feasible, and treatment should be performed in the case of rapid freezing disease examination during the operation. For the pulmonary nodules that are difficult to locate in the superficial subpleural surface, Preoperative positioning of hook wire and wire guided by $\mathrm{CT}$, injection of sclerotherapy or injection of methylene blue, and other methods were feasible. Rapid freezing examination was performed after intraoperative wedge resection. The advantages of wedge resection in superficial pulmonary nodules are that the operation is simple and rapid, and sufficient margin depth can be obtained. However, for deep pulmonary nodules that are difficult to locate, wedge resection is difficult to locate and the resection area is enlarged. Lobectomy is associated with greater loss of lung function, especially in patients with poor lung function and the elderly. Segmental pneumonectomy has the following advantages: it can dissect bronchus, arteries, and veins. The normal function of adjacent lung segments is not affected, and the lung function of patients can be preserved to the greatest extent; it avoids hemoptysis caused by obstruction of venous return due to the removal of veins in normal pulmonary segments during wedge resection. The expansion and collapse method was used to accurately cut the intersegment plane, reduce postoperative complications such as air leakage, and ensure enough incision margin, in line with the principles of oncology. The lung was in a dilated state and in a normal anatomical position during the three-dimensional reconstruction. However, in the thoracoscopic surgery, the operative side of the lung collapsed and was pulled from the front to the back and left to the right, so there was a slight difference in the direction of the segmental vessels and bronchus in the two different conditions. At this point, the surgeon needs to place the lung back to a relatively normal anatomical shape and accurately identify the tracheal vessels with 3D CTBA.

\section{Conclusions}

In conclusion, for deep pulmonary nodules or transsegmental pulmonary nodules, 3D CTBA is safe and feasible for thoracoscopic anatomical segmental pulmonary resection, which can not only ensure a safe incision margin but also preserve as much lung tissue as possible and fully preserve lung function. Therefore, 3D CTBA plays an important role in precision pulmonary segmentectomy.
Although the current results show that patients with early lung cancer have a longer survival after pneumonectomy. However, there is still an unavoidable fact that many patients cannot afford pneumonectomy, especially those with lung cancer or serious basic diseases. The wedge-shaped resection of a good spitter lesion cannot meet the needs of treatment, and the dissection of the dissection is obviously more appropriate. Thoracoscopic surgery is more advantageous than the chest surgery, and we must strive to make the pleural mirror lung segment to be accurate, safe, and conforming to the prototype of tumor treatment. This is the development trend of surgical treatment of lung cancer, which not only requires skilled surgical skills but also requires advanced technologies such as accurate, visual, threedimensional reconstruction images.

\section{Data Availability}

The data used to support the findings of this study are available from the corresponding author upon request.

\section{Conflicts of Interest}

The authors declare that they have no conflicts of interest.

\section{References}

[1] X. Zhao, B. Zhao, S. Yao, and K. Ding, "Clinical application of three-dimensional printing-assisted arthroscopic reconstruction of medial patellofemoral ligament to treat recurrent patellar dislocation in adolescents," Asian Journal of Surgery, vol. 43, no. 12, pp. 1191-1193, 2020.

[2] W. Xie, P. Gao, and L. Ji, "Three-dimensional spiral ct measurement of atlantal pedicle and its clinical application," Experimental and Therapeutic Medicine, vol. 14, no. 2, pp. 1467-1474, 2017.

[3] S. Gao, B. Qiu, L. Fang et al., "Comparison of thoracoscopic anatomical partial-lobectomy and thoracoscopic lobectomy on the patients with ptlan $0 \mathrm{~m} 0$ peripheral non-small cell lung cancer," Zhonghua Wai Ke Za Zhi, vol. 53, no. 10, pp. 727-730, 2015. 